Journal of Science and Technology, Vol. 32, No. 1 (2012), pp 32-41 32

(C) 2012 Kwame Nkrumah University of Science and Technology (KNUST)

http://dx.doi.org/10.4314/just.v32i1.5

\title{
RESEARCH PAPER \\ THE ENTROPY THEORETIC MEASURE FOR MANPOWER SYSTEMS IN PERSPECTIVE
}

\author{
V. U. Ekhosuehi and A. A. Osagiede \\ Department of Mathematics, University of Benin, Benin City, Nigeria \\ virtue.ekhosuehi@uniben.edu
}

\begin{abstract}
:
This study provides a realistic appraisal of the use of entropy measures in manpower systems. The appraisal shows that the existing entropy measures for manpower systems give a partial picture of the behavioural mechanism of the system. Consequent upon this, we propose the use of transition probabilities of the imbedded Markov chain for manpower systems as inputs in the entropy statistic. The proposal is illustrated by refining the basic Shannon entropy rate and implemented in Matlab computing environment.
\end{abstract}

Keywords: : entropy, manpower system, Markov chain, Matlab package.

\section{INTRODUCTION}

Manpower systems are well-known hierarchical systems in literature that consist of individual stocks and flows (Bartholomew et al., 1991; and McClean et al., 1992). As a consequence, a unified framework is employed in the study of manpower systems. Most authors rely on Markov chain methodologies as an analytic tool to unify the states of a manpower system with the axiomatic foundation that there is a onestage dependence of events, i.e. each event depends immediately on the preceding event, but not on the other prior events. The works of Feichtinger and Mehlmann (1976), Raghavendra (1991), Setlhare (2007) and Tsaklidis (1994) are just a few references. The approach by these aforementioned authors is enough driving force to assume that any model for manpower systems should unify the states of the system.

The implementation of Markovian manpower models (MMMs) is characterized by computational complexities. For this reason, several software packages such as KENT, PROSPECT, MICROPROSPECT, CAMPLAN, and MANSIM (Bartholomew et al., 1991; Smith and Bartholomew, 1988); and FORMASY (Verhoeven, 1981) have been developed to facilitate the use of MMMs. These packages are tailored towards specialized problems and thus cannot be easily modified. Ekhosuehi and Osagiede (2010a) employed the Matlab package as a computational tool in analyzing manpower systems. The suitability of Matlab for manpower systems emanates from the hierarchical nature of the system which is often represented in a matrix form. Matlab has several computational and 
graphic visual advantages arising from its interactive interface and independent plotting devices. Details on the essential features of Matlab are contained in Highman and Highman (2000), Martinez and Martinez (2002) and Hahn and Valentine (2010).

The term entropy refers to a measure of the degree of disorderliness, flexibility, uncertainty or randomness in a system (see Tirtiroglu, 2005). Entropy-theoretic methodologies have been applied to diverse and substantive areas such as: queuing systems (Tirtiroglu, 2005), external debt stock (Osagiede and Ekhosuehi, 2007), gender inequality (Ekhosuehi and Osagiede, 2010b), goodness-of-fit tests (Lee et al., 2011) and, in particular, to manpower systems (McClean and Abodunde, 1978). However, we shall primarily limit our scope of study to entropy-theoretic measures for manpower systems.

\section{MATERIALS AND METHODS} Some preliminaries to entropy-theoretic measures

The concept of entropy can be formalized from different perspectives. In statistical physics, entropy, denoted as $S$, is related logarithmically to the number of microstates, $W$, as

$S=k_{B}$ In $W$

where the constant $k_{B}$ could in principle be chosen arbitrarily; but for convenience, as Boltzmann constant (Kneen et al., 1972). Suppose $n_{0}, n_{1}, n_{2} \ldots$ (are the numbers of atoms with no energy quanta, 1 quantum, 2 quanta, and so on) represent the numbers of the energy levels, then the number of ways $W$ for the Boltzmann distribution of energy is

$W=\frac{N !}{n_{0} ! n_{1} ! \ldots n_{i} ! n_{j} ! \ldots}$

where $N$ is the total number of atoms in the system (Kneen et al., 1972). For two blocks, $W_{l}$ and $\mathrm{W}_{2}$, the number of ways of distributing energy at the instant of contact is given by Toda et al. (1978) as
$W=W_{1} \times W_{2}$

Frequently in chemistry, entropy changes are determined by making experimental (calorimetric) measurements on the system. The calorimetric measurement of entropy for an infinitesimal change is

$d s=\frac{d q}{T}$

where $d q$ is the quantity of heat and $T$ is the temperature. Kneen et al. (1972) reported that this calorimetric measurement form is the way in which the idea of entropy was introduced before a molecular understanding of the concept. To apply equation (4), we use

$\Delta S=\int_{1}^{2} \frac{d q}{T}$

where 1 and 2 denote the initial and final states, and $\Delta S=S_{2}-S_{1}$.

In information theory, the amount of information contained in a piece of data is quantified in Shannon entropy. The Shannon entropy function is used to measure message uncertainty and communication channel capacity. Shannon entropy relates the notion of information provided by a probability distribution for predicting outcomes to uncertainty and "choice" wherein a source transmits discrete signals $\mathrm{X}=\left\{x_{1}, x_{2}, \ldots, x_{n}\right\}$ through a noiseless channel according to a probability distribution .

$\Delta_{n}=\left\{\left(p_{1}, p_{2}, \ldots p_{n}\right): p_{i}=p\left(x_{i}\right) \geq 0, \sum_{i=1}^{n} p_{i}=1\right\}$

The uncertainty of the system is measured by $U_{i}=-\log _{b}\left(p\left(x_{i}\right)\right)$, where $b$ is the base of the logarithm. Logarithm is used here to provide the additive characteristic for uncertainty. The lower the probability $p\left(x_{i}\right)$ i.e. $p\left(x_{i}\right) \rightarrow 0$ the higher the uncertainty i.e. $\mathrm{Ui} \rightarrow \infty$, for the outcome $x_{l}$. The average uncertainty $\langle U\rangle$, with $\langle\bullet\rangle$ being the average operator is given as ... 
$\langle U\rangle=\sum_{i=1}^{n} p\left(x_{i}\right) U_{i}=-\sum_{i=1}^{n} p\left(x_{i}\right) \log _{b} p\left(x_{i}\right)$

Equation (6) is referred to as Shannon entropy (Ciuperca and Girardin, 2005). According to Ciuperca and Girardin (2005), entropy rate was first defined by Shannon for an ergodic Markov chain with finite state space $E$ as the sum of the entropies of the transition probabilities $\left(p_{i j}\right), \mathrm{j}=$ $1,2, \ldots, s$ weighted by the probability of occurrence of each state $i$ according to the stationary distribution éa of the chain, namely

$H(X)=-\sum_{i=1}^{s} \pi_{i} \sum_{j=1}^{s} p_{i j} \log p_{i j}$

Shannon entropy has the property of symmetry i.e. the measure is unchanged if the outcomes $x_{i}$ are re-ordered, and it increases with the number of outcomes i.e.

$H_{n}\left(p_{1}, \ldots, p_{n}\right) \leq H_{n}\left(\frac{1}{n}, \ldots, \frac{1}{n}\right)<H_{n+1}\left(\frac{1}{n+1}, \ldots \frac{1}{n+1}\right)$

where $H_{n}(\bullet)$ is the entropy measure. With Shannon formula the entropy of a system can be calculated from the entropy of its sub-systems if we know how the sub-systems interact with each other. For instance, for positive integers $b_{i}$ where $b_{i}+\ldots+b_{k}=n$, we have

$H_{n}\left(\frac{1}{n}, \ldots, \frac{1}{n}\right)=H_{k}\left(\frac{b_{1}}{n}, \ldots \frac{b_{k}}{n}\right)+\sum_{i=1}^{k} \frac{b_{i}}{n} H_{b_{i}}\left(\frac{1}{b_{i}}, \ldots \frac{1}{b_{i}}\right)$

In most applications, Shannon entropy is preferred to other entropy measures because it is less mathematically rigorous than either thermodynamic entropy or Boltzmann's entropy, and it relies solely on probabilities rather than on causal statements. Further details on Shannon entropy are found in the publication: Departmento de Matematica (2001).

\section{Extension of the information-theoretic en- tropy measure}

Girardin and Limnios (2003) expressed Shannon entropy for a finite measure $\tilde{a}^{G}$ absolutely continuous with respect to a $\sigma$-finite measure ã $=$ with Radon-Nikodym derivative $f$ as

$S_{\mu}(f)=-\int f(x) \log f(x) d \mu(x)$

For a discrete measure ã ${ }^{G}$ with support $E$,

$S\left(\mu^{*}\right)=-\sum_{i \in E} \mu_{i}^{*} \log \mu_{i}^{*}$

For a discrete-time process $X=\left(X_{n}\right)_{n j N}$, the entropy at time $n$ is defined as the Shannon entropy of the $n$-dimensional marginal distribution of $X$, namely

$H_{n}(X)=-E\left[\log f_{n}^{X}(X)\right]=S_{\mu_{n}}\left(f_{n}^{X}\right)$

where $f_{n}^{x}$ is the density function of the random vector $X=\left(X_{1}, X_{2}, \ldots, X_{n}\right)$ with respect to some reference measure $\tilde{a}_{n}$.

Barron (1985) proved that if $\left\{X_{n}\right\}$ is a stationary ergodic process with probability densities $f\left(X_{l}\right.$, $\left.X_{2}, \ldots, X_{n}\right)$, then the sequence of relative entropy densities $(1 / n) \log f\left(X_{1}, X_{2}, \ldots, X_{n}\right)$ converges almost surely to the relative entropy rate, and to the shift invariant random variable $E \log f$ $\left(\mathrm{X}_{0} \mid \mathrm{X}_{-\infty,-1}\right)$, for nonergodic processes. Barron (1986) expressed Shannon entropy in terms of normal entropy and $D(X)$ as:

$H=(1 / 2) \log 2 \pi e \sigma^{2}-D(X)$

where $\quad D(X)=\int \frac{f_{n}(x) \log f_{n}(x)}{\phi(x)} d x$

$f_{n}(x)$ is a probability density function, $\left.\tilde{\mathrm{N}} \neq x\right)$ is the normal density function, and $\sigma^{2}$ is the variance.

Girardin and Limnios (2003) obtained the entropy rate of semi-Markov processes by extending the Shannon-McMillan-Breiman theorem. The Shannon-McMillan-Breiman theorem (or asymptotic equirepartition property (AEP)) constitutes the analogue of the law of large numbers for information theory. This theorem 


\section{5}

Ekhosuehi and Osagiede

ensures the almost sure convergence of the sequence $-\left(\log f_{n}^{X}(X)\right)$ to $H(X)$, where $f_{n}^{X}(X)$ is the density function of the random vector $X=\left(X_{1}, \ldots, X_{n}\right)$ and $H(X)$ is the entropy rate. Since semi-Markov processes belong to a class of non-stationary continuous-time processes, Girardin and Limnios (2003) presented the entropy of the process $\mathrm{Z}$ as

$-\frac{1}{T} \log f_{T}^{Z}(Z) \rightarrow H(Z)$

Ciuperca and Girardin (2005) derived an estimator for the entropy rate based on the maximum likelihood method of the transition matrix with proofs of its properties. The entropy rate is of the form

$H(X)=\sum_{i=1}^{s} \pi_{i} \log \pi_{i}-\sum_{i=1}^{s} \sum_{j=1}^{s} \pi_{(i, j)} \log \pi_{(i, j)}$

where $X=\left(X_{n}\right)$ is a homogeneous ergodic (that is irreducible and aperiodic) Markov chain with finite state space $E=\{1, \cdots, s\}, \pi_{i}, i=1, \cdots, s$, is the stationary distribution, $\pi(i, j)=\pi_{i} P_{i j}$ and $P_{i j}=P\left(X_{\mathrm{n}}=j / X_{n-l}=i\right), n P_{i j} \geq 1$. The stationary distribution $\left(\pi_{i}\right)$ is estimated as

$\hat{\pi}_{i}=\frac{N_{n}(i)}{n}, i, j=1, \ldots, s$, where

$N_{n}(i)=\sum_{m=1}^{n} N_{n}(i, j) \stackrel{\text { and }}{ } N_{n}(i, j)=\sum_{m=1}^{n} 1_{\left\{X_{m-1}=i, X_{m}=j\right\}}$

for the one observation of the chain, $\left(X=X_{0, \ldots .,} X_{n}\right)$.

$\stackrel{\wedge}{\text { a.s. }} \rightarrow \pi_{i}$ and $\sqrt{n}\left(\hat{\pi}_{i}-\pi_{i}\right) \stackrel{L}{\rightarrow} N\left(0, \pi_{i}\left(1-\pi_{i}\right)\right)$ $\mathrm{C}$ i u -

perca and Girardin (2005) obtained an estimate of the stationary probability for given $K$ independent observations of the chain,

$X^{(k)}=\left(X_{0}^{(k)}, \ldots, X_{n}^{(k)}\right), k=1, \ldots, K$, for a fixed integer $n$ as $\hat{\pi}_{i}=\frac{n_{k}(i)}{K}, i, j=1, \ldots, s$, where

$n_{k}(i)=\sum_{k=1}^{K} 1_{\left\{X_{0}^{(k)}=i\right\}}$ and $\hat{\pi_{i}} \stackrel{\text { a.s. }}{\rightarrow} \pi_{i}$ and

$\sqrt{K}\left(\hat{\pi}_{i}-\pi_{i}\right) \stackrel{L}{\rightarrow} N\left(0, \pi_{i}\left(1-\pi_{i}\right)\right)$

Application of entropy-theoretic methodologies to manpower systems

The use of entropy-theoretic concepts in manpower systems has produced interesting results. McClean and Abodunde (1978) resolved one of the problems associated with Shannon entropy (which is the absence of a fixed maximum value) by modifying the basic Shannon entropy so that it lies within the closed interval $[0,1]$ as

$H=-\frac{\sum_{i=1}^{k} p_{i} \log p_{i}}{\log k}$

for a steady-state manpower system. In equation (15), $k$ is the maximum number of tenure classes; and $p_{i}$ is the probability that a member of staff is in tenure class $i$. McClean and Abodunde (1978) used the transition probabilities generated from the McClean's steady-state model (McClean, 1977) as inputs to the formula in equation (15) so as to measure the stability of the length of service in a system. The work of McClean and Abodunde (1978) has been extended by Vassiliou (1984) to find the probability of person in a specific length of service class as $t \rightarrow \infty$.

Omosigho and Osagiede (1999) estimated the entropy value for an organisation based on wastage rate. The log-normal model of Chu and Lin (1994) was modified as

$W_{i}^{*}(t)=W_{i}(t)+L_{i}$

where 
$W(t)=\frac{1}{\sigma \sqrt{2 \pi}} \exp \left\{-\frac{1}{2}\left(\frac{\ln t-w}{\sigma}\right)^{2}\right\}, t \geq 0$

$L_{i}=\frac{W_{i}(t)}{\sum_{i=1}^{n} W_{i}(t)}-W_{i}(t)$

$W(t)$ is the wastage rate when the length of service is $t, w$ is the mean of $1 \mathrm{n} t$, and $\sigma$ is the standard deviation of $1 \mathrm{n} t$. Using the Bowey's stability curve for the actual wastage rates, Omosigho and Osagiede (1999) validated the entropy value for the organisation.

McClean (1986) developed a new definition of entropy for a manpower system based on continuous-time tenure profile as

$H^{*}=\frac{-\left(\int_{0}^{\infty} \frac{F(x)}{\mu} \log \frac{F(x)}{\mu} d x\right)}{\log w}$

where $F(x)=$ the survivor function, $\mu=\int_{0}^{\infty} F(x) d x$

and $w$ is the maximum possible completed length of service (C. L. S.). The entropy measure in McClean (1986) was aimed at correcting the anomaly of 'time-based drift' of the discrete entropy definition in McClean and Abodunde (1978). The crux of the entropy measure in McClean (1986) is the survivor function and the assumption that the modelled manpower system is in a steady-state. The limitations in the use of McClean's entropy measure are that the tail of the distribution of the survivor function is frequently ignored (Tyler, 1986) and that large statistical samples covering a short time span are required to achieve the steady-state property. Ebrahimi et al. (2010) had also mentioned that the information methodologies are often developed in isolation, i.e. a particular measure is used without consideration of the larger picture.

In Omosigho and Osagiede (2002) the assump- tion of a constant workforce size in the McClean/Abodunde's entropy measure was criticized as unrealistic. Thus, Omosigho and Osagiede (2002) introduced the augmented ratio of squares measure given as $f(x)=\frac{x^{2}}{1+x^{2}}$,

where $x^{2}=\sum_{j=1}^{k}\left(\frac{E_{j}-O_{j}}{E_{j}}\right)^{2}$,

$k$ is the highest grade in the organization, $E_{j}$ is the expected number of workers in grade $j$ in the organization and $O_{j}$ is the observed number of workers in grade $j$ in the organization. Omosigho and Osagiede (2002) therefore recalibrated $f(x)$ so as to detect the development of a chaotic situation in manpower systems as

$F(x)=\frac{1+k}{k} f(x)$

A stable and desirable workforce is indicated when $F(x)=0$, whereas $F(x)=1$ indicates an abnormal situation. For $F(x)>1$, a chaotic situation develops in the workforce of the organization. The stability measures proposed by Omosigho and Osagiede (2002) have some limitations such as:

i. The measures do not take into consideration the transition of individuals from one grade/cadre to another.

ii. The range of $f(x)$, i.e. $f(x) \in[0,1]$, is unrea istic in practice as the highest grade in any organization, $k$, is known and finite. Thus $k \rightarrow \infty$ as claimed by Omosigho and Osagiede (2002) cannot hold in practice.

Tyler (1989) developed an entropy measure for manpower systems based on the concepts of thermodynamics. Tyler (1989) assumed that the size of the manpower system is analogous to the absolute temperature and that the size of the tenure class satisfies the McClean's steadystate manpower model. Tyler (1989) evaluated the entropy of a manpower system from the 


\section{Ekhosuehi and Osagiede}

Boltzmann's formula as well as the internal energy of the manpower system using

$S=\frac{H_{B}}{\log c}$

where $H_{B}$ is the Boltzmann's formula expressed as

$H_{B}=\log \left\{\frac{N !}{N_{1} ! N_{2} ! \ldots N_{c} !}\right\}$

$N_{1}=N_{1}, N_{k}=N_{1} \prod_{i=1}^{k-1} p_{i}, k>1$

are the McCleans' steady-state number of members of the population belonging to each tenure class $c, c$ is the number of tenure classes, $N=\sum_{i=1}^{c} N_{i}$ is the total

size of the population, and $p_{i}$ is the probability of surviving from one tenure class $i$ to tenure class $i+1$. The thermodynamic entropy measure proposed by Tyler (1989) has a critical limitation emanating from the assumption that the size of the manpower system is analogous to absolute temperature.

This assumption is unrealistic as it has no theoretical basis. More so, the mathematical complexities inherent in the application of the entropy measure limit its use only to the mathematically sophisticated researcher.

\section{RESULTS AND DISCUSSION}

Suppose we are interested in estimating the entropy value of a manpower system described by the following transition mechanism:

$\mathbf{P}_{0}=\left[\begin{array}{lllllll}0.8872 & 0.1128 & 0 & 0 & 0 & 0\end{array}\right]$,

where $\mathbf{P}_{0}$ is the recruitment vector, $\mathbf{P}$ is the
$\mathbf{P}=\left[\begin{array}{cccccc}0 & 0.9891 & 0 & 0 & 0 & 0 \\ 0 & 0 & 0.9551 & 0 & 0 & 0 \\ 0 & 0 & 0 & 0.9636 & 0 & 0 \\ 0 & 0 & 0 & 0 & 0.9724 & 0 \\ 0 & 0 & 0 & 0 & 0 & 0.9102 \\ 0 & 0 & 0 & 0 & 0 & 0.3891\end{array}\right]$

and

$\mathbf{w}=\left[\begin{array}{l}0.0109 \\ 0.0449 \\ 0.0364 \\ 0.0276 \\ 0.0898 \\ 0.6109\end{array}\right]$

transition probability matrix, and $\mathbf{W}$ is the wastage probability vector. The maximum number of grades in the system is the total number of the states in the transition matrix, i.e. $k=6$.

If we decide to apply the formula in equation (15) to estimate the entropy of the system, then we consider the survival of individuals from one grade to the other as described by the transition matrix, P. Now, for a direct transition from grade 1 to other grades, we have $p_{1}=0$, $p_{2}=0.9891$, and $p_{1}=0, i=3,4,5,6$. In this case,

we find that the axiom that $\sum_{i=1}^{6} p_{i}=1$ does

not hold. The same result holds if the other grades are chosen as a starting point. The reason for this is that the transition matrix $\mathbf{P}$ is sub -stochastic as a result of wastages in the system. Thus, we cannot use the entropy measure in McClean and Abodunde (1978) as well as its variants in Vassiliou (1984), Omosigho and Osagiede (1999) and Omosigho and Osagiede 
(2002) to do this because flows such as recruitment and wastage flows are neglected. Consequently, the entropy measures in McClean and Abodunde (1978), Vassiliou (1984), Omosigho and Osagiede (1999) and Omosigho and Osagiede (2002) give a partial picture of the behaviour of the system. In a similar manner, the thermodynamic entropy measure in Tyler (1989) cannot be applied because there is no sufficient reason to assume that the size of the manpower system is analogous to absolute temperature. Similarly, if we decide to use the basic Shannon entropy rate or the entropy formula of Ciuperca and Girardin (2005), we again encounter the problem of dealing with a substochastic transition matrix

a s $\sum_{j=1}^{k} p_{i j} \leq 1$

where the shortfalls in the sum of transition probabilities, are created by losses and transfers in the system, and $p_{i j}$ is the transition probability from state $i$ to state $j$. Since the states within

the system are transient, then $\lim _{n \rightarrow \infty} p_{i j}^{(n)}=0$

for all $i$ (see Hillier and Lieberman, 2005). This is because the process cannot remain in a transient state after a large number of transitions. To compute the entropy value for the system therefore, a transformation of the form: $I:\left\langle\mathbf{P}, \mathbf{w}, \mathbf{P}_{\mathbf{0}}\right\rangle \rightarrow k \times k$ matrix space is necessary. The imbedded Markov chain formulation which is based on the Young/Almond-type transition matrix (Feichtinger and Mehlmann, 1976; Tsaklidis, 1994) is a convenient way to achieve this. By so doing, we seek a block matrix of the form: $\mathbf{Q}=\mathbf{P}+\mathbf{w} \mathbf{P}_{\mathbf{0}}$, such that $\mathbf{Q e}^{\prime}=\mathbf{e}^{\prime}$, where $\mathbf{e}^{\mathbf{\prime}}$ is a column vector conformable with matrix $\mathbf{Q}$ containing one as its elements. In this light, we modify the basic Shannon entropy rate using the transition probabilities of the imbedded Markov chain as inputs in the Shannon entropy formula and $\log _{\mathrm{e}} k$ as a scaling factor to constrain the entropy rate to the interval $[0,1]$. The modified version of the basic Shannon entropy rate for the manpower system under consideration is written in Matlab pseudocodes as:

$$
\begin{aligned}
& \mathrm{k} \leftarrow 6 ; \mathrm{Q} 0 \leftarrow \mathbf{P}+\mathbf{w}^{*} \mathbf{P}_{0}, \\
& \mathrm{PI} \leftarrow \operatorname{diag}\left(\mathrm{Q}^{\wedge} 100\right)
\end{aligned}
$$

Modified_s hannon_ent ropy $\leftarrow$

- sum $\left(\left(\mathrm{PI}{ }^{*} \log (\mathrm{Q})\right)\right) / \log (\mathrm{k}){ }^{\prime}$

where the left arrow $\leftarrow$ is used instead of equality for an assignment and $\mathrm{Q}$ is a matrix formed from Q0 such that the zeros in matrix Q0 are replaced by 1 . We implement the program described by the pseudocodes in Matlab R2007b. Subsequently, we obtain the imbedded Markov chain and the entropy value for the manpower system respectively as

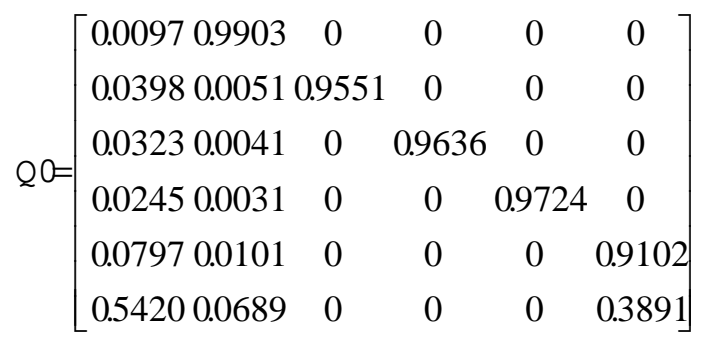

and

Modified_s hannon_ent ropy $=0.1871$

In matrix Q0, the entries in columns 1 and 2 arise from the replacement matrix and the recruitment policy of the system as new recruits enter either into grade 1 or grade 2 . In particular, the $\mathbf{1 , 2}$ entry is the probability that an individual is promoted from grade 1 to grade 2 or he is recruited into grade 2 to replace leavers in grade 1 . Wherever zero entry occurs, it means no transition took place between the corresponding grades. The main diagonal elements of matrix Q0 are either zero or relatively small, while the upper off-diagonal elements ('promotion' probabilities) are large. The main diagonal elements for columns 1 and 2 show the consequential replacement of losses by new 
recruits, while the upper off-diagonal elements indicate that there is a normal progression to the next higher grade. The entropy value, which is closer to zero than to one, indicates that uncertainty in the transition mechanism of the system is low. This inference is what we expect as the transition probability matrix $\mathbf{P}=\left(p_{i j}\right)$ is such that $p_{i j} \geq 0$, for $j=i, i+1$, and $p_{i j}=0$, otherwise; and the imbedded Markov chain Q0 has a 'sparse' block structure. In view of these, an individual in the system is almost sure of the next grade in the immediate ensuing transition.

From this illustrative example, we are able to make the Shannon entropy rate robust using the imbedding transformation to unify the plethora of transitions in the system.

\section{CONCLUSION}

This study contains a survey of entropytheoretic literature with particular reference to manpower systems. Although the existing entropy measures give a partial picture of the realworld intricacies of a typical manpower system and thus limit their use in describing the entropy of the entire system, yet the study does not invalidate the use of entropy in manpower systems. The major accomplishments of the study are that entropy as a measure of certain properties in manpower systems has been appraised to identify future research directions, and arising thereof, is a suggestion for the development of a robust entropy statistic in which all possible transitions in a manpower system are inscribed. The suggestion is substantiated with an example problem.

\section{ACKNOWLEDGEMENT}

We acknowledge the constructive suggestions of the anonymous reviewers of the manuscript which have led to the improved quality of this paper.

\section{REFERENCES}

Bartholomew, D. J., Forbes, A. F. and McClean, S. I. (1991). Statistical techniques for manpower planning ( $2^{\text {nd }}$ ed.). John Willey \& Sons: Chichester.
Barron, A. R. (1985). The strong ergodic theorem for densities: Generalised ShannonMcMillan-Breiman theorem, The Annals of Probability, 13 (4): 1292-1303.

Barron, A. R. (1986). Entropy and the central limit theorem, The Annals of Probability 14 (1):336-342.

Chu, S. C. K. and Lin, C. K. Y. (1994). Cohort analysis technique for long-term manpowerplanning: the case of a Hong Kong tertiary institution, Journal of OperationalResearch Society, 45(6): 696-709.

Ciuperca, G. and Girardin, V. (2005). On the estimation of the entropy rate of finite Markov chains. Retrieved 24/05/2009 from http://conferences.telecom-bretagne.eu/ asmda2005/IMG/pdf/proceedings/1109.pdf

Departmento de Matematica (2001). Measures of uncertainty: Shannon's entropy. Retrieved 22/05/2009 from www.mtm.ufsc.br/ taneja/ book/node3.html.

Ebrahimi, N., Soofi, E. S. and Soyer, R. (2010). Information measures in perspective, International Statistical Review, 78 (3): 383-412.

Ekhosuehi, V. U. and Osagiede, A. A. (2010a). Enrolment transition process with MATLAB as a technical computing language, African Journal of Contemporary Issues, 10 (1):151163.

Ekhosuehi, V. U. and Osagiede, A. A. (2010b). The use of entropy index for gender inequality analysis, Global Journal of Mathematical Sciences, 9 (1): 25-30.

Feichtinger, G. and Mehlmann, A. (1976). The recruitment trajectory corresponding to particular stock sequences in Markovian personflow models, Mathematics of Operation Research, 1(2): 175-184.

Girardin, V. and Limnios, N. (2003). On the 
entropy for semi-Markov processes, Journal of Applied Probability, 40: 1060-1068.

Hahn, B. and Valentine, D. (2010). Essential $M A T L A B$ for engineers and scientists $\left(4^{\text {th }}\right.$ ed.)Elsevier Ltd., UK.

Higham, D. J. and Higham, N. J. (2000). MAT$L A B$ guide. Society for Industrial and AppliedMathematics (SIAM), Philadelphia.

Kneen, W. R., Rogers, M. J. W. and Simpson, P. (1972). Chemistry: Facts, patterns, and principles. Addison-Wesley Publishers Ltd, London.

Lee, S., Vonta, I. and Karagrigoriou, A. (2011). A maximum entropy type test of fit, Computational Statistics and Data Analysis, 55 (9):

Martinez, W. L. and Martinez, A. R. (2002). Computational statistics handbook withMAT$L A B$. Chapman \& Hall/CRC, Florida.

McClean, S. (1977). The steady-state behaviour of a manpower planning model in which class corresponds to length of service, $O p$ erational Research Quarterly, 28 (2i):305311.

McClean, S. and Abodunde, T. (1978). Entropy as a measure of stability in manpower system. Journal of Operational Research Society, 29(9); 885-889.

McClean, S., Lundy, P. and Ewart, W. (1992). Predicting the growth of manpower for the Northern Ireland Software Industry, Journal of the Royal Statistical Society. Series D (The Statistician), 41: 349-356. 2635-2643.

Workforce stability and suitability, Journal of the Nigerian Association of Mathematical Physics, 6: 333-339.

Osagiede, A. A. and Ekhosuehi, V. U. (2007). Extending entropy stability measure to external debt structure, Journal of Science and Technology, 27 (3):156-162.

Raghavendra, B. G. (1991). A bivariate model for Markov manpower planning systems, The Journal of the Operational Research Society, 42 (7): 565-570.

Setlhare, K. (2007). Optimization and estimation study of manpower planning models. PhDThesis, University of Pretoria, South Africa.

Smith, A. R. and Bartholomew, D. J. (1988). Manpower planning in the United Kingdom: an historical review. The Journal of the Operational Research Society, 39 (3): 235-248.

Tirtiroglu, E. (2005). An entropy measure of operating performance uncertainty in queues: Markovian examples. International Journal of Operational Research, 1(1\&2): 204-212.

Toda, M., Kubo, R. and Saito, N. (1978) Statistical physics I: Equilibrium statistical mechanics ( $2^{\text {nd }}$ ed.). Springer-Verlag: New York.

Tsaklidis, G. M. (1994). The evolution of the attainable structures of a homogeneous Markov system with fixed size. Journal of Applied Probability, 31 (2): 348-361.

Tyler, G. W. (1986). Organization size and staff tenure. Journal of Operational Research Society, 37(4): 373-380.

Omosigho, S. E. and Osagiede, A. A. (1999). Tyler, G. W. (1989). A thermodynamic model of On estimation of entropy value for an organisation, Journal of the Nigerian Association of Mathematical Physics, 3:36-42. manpower systems. Journal of Operational Research Society, 40(2): 137-139.

Vassiliou, P. C. G. (1984). Entropy as a measure of the experience distribution in a manpower

Omosigho, S. E. and Osagiede, A. A. (2002). 


\section{Ekhosuehi and Osagiede}

system. Journal of Operational Research Society, 35(11): 1021-1025.

Verhoeven, K. J. (1981). Corporate manpower planning. European Journal of Operational Research, 7: 341-349. 